\title{
A Facile Modifier-free Approach to Fabricate Antistatic Superhydrophobic Composite Coatings with Remarkable Thermal Stability and Corrosion Resistance
}

\author{
Xiang Liu ${ }^{1,2}$, Dekun Zhang ${ }^{3 *}$, Zhiguang Guo ${ }^{2,4 *}$ \\ 1. School of Mechatronic Engineering, China University of Mining and Technology, Xuzhou 221116, China \\ 2. State Key Laboratory of Solid Lubrication, Lanzhou Institute of Chemical Physics, Chinese Academy of Sciences, \\ Lanzhou 730000, China \\ 3. School of Materials Science and Engineering, China University of Mining and Technology, Xuzhou 221116, China \\ 4. Ministry of Education Key Laboratory for the Green Preparation and Application of Functional Materials, \\ Hubei University, Wuhan 430062, China
}

\begin{abstract}
Research on antistatic superhydrophobic surfaces has attracted widespread attention in some fields. However, in the application of superhydrophobic materials, fabricating stable and practical superhydrophobic surfaces through facile and low-cost approaches still faces considerable challenges. Herein, a polyphenylene sulfide (PPS)-based antistatic superhydrophobic composite coating with a high water contact angle $\left(166^{\circ}\right)$ and a low sliding angle $\left(2^{\circ}\right)$ was fabricated on a Q345 steel surface through a simple spray-coating method without any modifier. Furthermore, the as-prepared superhydrophobic coating also displayed excellent superhydrophobicity for water droplets at different $\mathrm{pH}$ values, as well as self-cleaning, anti-fouling and anti-icing properties. Importantly, the superhydrophobic coating still exhibited superhydrophobicity after calcination at $350{ }^{\circ} \mathrm{C}$ for $1 \mathrm{~h}$, indicating its outstanding thermal stability. Excellent antistatic and anticorrosion properties were obtained on the prepared coating surface, which allows the coating to be applied under harsh conditions. Benefiting from the above characteristics, compared with the commercial coating, the as-obtained antistatic superhydrophobic coating may be applied more widely in related fields.
\end{abstract}

Keywords: no-modifier, superhydrophobicity, thermal stability, antistatic property, anticorrosion

Copyright $(0)$ The author(s) 2020 .

\section{Introduction}

Metal materials are widely used in various fields because of their excellent comprehensive properties ${ }^{[1]}$. Nevertheless, poor corrosion resistance seriously affects their service life and limits their further applications ${ }^{[2]}$. It is well known that a surface with superhydrophobicity can play a good protective role on the matrix. Superhydrophobic surfaces, inspired by plants and animals in nature, show significant importance in fundamental research and industrial applications such as corrosion-resistance $^{[3-5]}$, self-cleaning ${ }^{[6,7]}$, anti-icing ${ }^{[8-10]}$, and drag reduction ${ }^{[11,12]}$. They possess static contact angles of Water Contact Angles (WCAs) higher than $150^{\circ}$ and Sliding Angles (SAs) lower than $10^{\circ[4-6]}$. Various methods, such as sol-gel ${ }^{[13-15]}$, template ${ }^{[16,17]}$, self-assembly ${ }^{[18,19]}$ and etching ${ }^{[20-22]}$, have been adopted to fabricate superhydrophobic surfaces.

Coating is an important method for surface modification of materials ${ }^{[23]}$. Furthermore, spraying, which is not limited by the shape and size of the sample surface, is a simple and efficient surface treatment $\operatorname{method}^{[24]}$. In some special application fields, such as coal mining, aviation, defence and petrochemical applications, the coating should also possess excellent antistatic property and the surface resistance is usually less than $106 \Omega^{[25,26]}$. In recent years, with the development of science and technology, multifunctional superhydrophobic surfaces with outstanding antistatic performance have become a research focus in the superhydrophobic field ${ }^{[27,28]}$. Owing to the excellent electrical properties, electrostatic charges cannot

*Corresponding author: Zhiguang Guo, Dekun Zhang

E-mail: zguo@licp.cas.cn, dkzhang@cumt.edu.cn 
accumulate on the antistatic superhydrophobic surface, thus avoiding the hazards caused by electrostatic accumulation in special application fields ${ }^{[29]}$. Furthermore, antistatic superhydrophobic coatings also play an important role in waterproof electronic devices and electromagnetic interference shielding ${ }^{[30,31]}$. Carbon-based materials are often used to prepare conductive coatings because of their excellent conductivity. Currently, a series of carbon-based conductive superhydrophobic coatings have been obtained $^{[32-34]}$. For instance, Das et al ${ }^{\left[{ }^{[32]}\right.}$ fabricated a highly conductive superhydrophobic composite coatings filled with carbon nanofiber/PTFE polymer, which is the first study to report data on the effectiveness of conductive superhydrophobic composite coatings as ElectroMagnetic Interference (EMI) shielding materials. By partially embedding fluorinated graphene into ThermoPlastic Polyurethane (TPU), Wang et al. ${ }^{[33]}$ proposed a dissolution and re-solidification method to manufacture a superhydrophobic composite with highly stretchable and excellent electromechanical properties. Yao et al. ${ }^{[34]}$ employed a simple spraying method to prepare superhydrophobic nanocomposite coatings, composed of spherical $\mathrm{SiO}_{2}$ nanoparticles and fluorinated Multi-Walled Carbon Nanotubes (MWCNTs). The obtained coatings display fine transparency and conductivity. However, most of the reported surfaces were either modified with low surface energy modifiers or based on complex preparation processes, seriously hindering their further applications in practice. In addition, these surfaces display poor thermally stable superhydrophobicity (below $200{ }^{\circ} \mathrm{C}$ ), limiting the engineering value of them. Thus, it is more feasible to develop conductive superhydrophobic surfaces with good thermal stability by economical and widely available methods.

As a result of excellent conductivity and chemical stability, CNFs are widely applied in industrial production ${ }^{[35,36]}$. Rare earth oxides have been reported to show intrinsical hydrophobicity and still maintain stable hydrophobicity at $1000{ }^{\circ} \mathrm{C}^{[37]}$. Cerium dioxide $\left(\mathrm{CeO}_{2}\right)$, a widely used rare earth oxide, has been attracted intensive interest owing to its excellent properties ${ }^{[38]}$. Thus three-dimensional $\mathrm{CeO}_{2}$ particulates can not only provide rough structural support for superhydrophobic coatings, but also improve the thermally stable superhydrophobicity of the coatings. Additionally, polyphenylene sulfide (PPS), as a special engineering plastic, possesses excellent physical and chemical properties, including high flame retardancy, radiation resistance, good thermal stability and excellent electrical properties $^{[39]}$. As a coating, PPS has good bonding properties to the surface of carbon steel, cast iron, aluminum, glass and ceramics ${ }^{[40]}$. With excellent chemical stability, corrosion resistance and low surface energy, polytetrafluoroethylene (PTFE) is an ideal candidate material for surface modification ${ }^{[41]}$. Some studies related to PPS coatings by blending PTFE have been applied to prepare superhydrophobic surfaces ${ }^{[42-44]}$. Up to date, to our best aware, there are few reports on antistatic PPS/PTFE superhydrophobic composite coatings. It is vital and challenging task to fabricate antistatic superhydrophobic coatings with good thermal stability in industrial applications.

Herein, a simple, modifier-free and efficient method is applied to prepare antistatic superhydrophobic coatings with good thermal stability on the surface of alloy steel. The technology involved combining mechanical mixing and spraying, which is potentially applicable to the large-scale fabrication of conductive superhydrophobic coatings. Using this simple method, $\mathrm{CeO}_{2}$ particulates were fabricated by an oil bath method, and the surface roughness was achieved by blending $\mathrm{CeO}_{2}$ particulates and modified CNFs. Afterwards, a certain amount of PPS, PTFE and PDMS were added to the above mixed particles to generate a grey suspension, which could be used for spraying on the surface of alloy steel. The prepared coating, with a high WCA $\left(166^{\circ}\right)$ and low SA $\left(2^{\circ}\right)$, exhibited excellent hydrophobic properties. Furthermore, the superhydrophobic coating also displayed great antistatic property, corrosion resistance, thermal stability, fouling resistance and freezing resistance. It is believed that the processes elaborated in this article possess practical certain application value and reference significance for superhydrophobic research.

\section{Experiment}

\subsection{Materials}

Q345 steel sheets $(30 \mathrm{~mm} \times 20 \mathrm{~mm} \times 0.2 \mathrm{~mm})$ were purchased from Shanghai Baoshan Iron \& Steel Co., Ltd. 
CNFs $100 \mathrm{~nm}$ in diameter and $20 \mu \mathrm{m}-200 \mu \mathrm{m}$ in length were obtained from Sigma-Aldrich Co., Ltd. Cerous nitrate $\left(\mathrm{Ce}\left(\mathrm{NO}_{3}\right)_{3} \cdot 6 \mathrm{H}_{2} \mathrm{O}\right)$ was supplied by Chengdu Kelong Chemical Reagent Factory. Urea $\left(\mathrm{CO}\left(\mathrm{NH}_{2}\right)_{2}\right.$, 99.0\%) was provided by Shantou Xilong Chemical Co., Ltd. Tetrapropylammonium bromide (TPAB) was obtained from Sinopharm Chemical Reagent Co., Ltd. Poly (1,4-phenylene sulfide) (PPS, average Mn: $1 \times 10^{4}$, yellow powder) was purchased from Sigma-Aldrich Co., Ltd. PTFE powder (500 mesh - 600 mesh) was supplied by Shanghai Weiwei Plasticizing Co., Ltd. Polydimethylsiloxane (PDMS) was provided by Dow Silicones Corporation in the USA. The other reagents were used as received without further purification.

\subsection{Preparation of 3D flowerlike $\mathrm{CeO}_{2}$ particulates}

The $3 \mathrm{D}$ flowerlike $\mathrm{CeO}_{2}$ particulates were synthesized with a simple procedure based on previous literature ${ }^{[45]}$. In short, $3.7 \mathrm{~g} \mathrm{Ce}\left(\mathrm{NO}_{3}\right)_{3} \cdot 6 \mathrm{H}_{2} \mathrm{O}, 4.5 \mathrm{~g}$ $\mathrm{CO}\left(\mathrm{NH}_{2}\right)_{2}$ and $10.0 \mathrm{~g}$ TPAB were added into a round flask containing $300 \mathrm{~mL}$ ethylene glycol. Afterwards, the above mixed solution was magnetically stirred at $180{ }^{\circ} \mathrm{C}$ for $1 \mathrm{~h}$. After that, the $\mathrm{CeO}_{2}$ precursor was centrifuged at a speed of $6000 \mathrm{rpm}$ for $5 \mathrm{~min}$ and washed with ethanol three times. Finally, $\mathrm{CeO}_{2}$ particulates were obtained by calcining the precursor at $500{ }^{\circ} \mathrm{C}$ for $2 \mathrm{~h}$.

\subsection{Preparation of sodium dodecylbenzene sulfonate modified CNFs}

To enhance the dispersion of pristine CNFs in solution, sodium dodecylbenzene sulfonate (SDBS) was used to modify carbon nanofibers, denoted as SDBS-CNFs. Briefly, $0.3 \mathrm{~g}$ CNFs and $0.3 \mathrm{~g}$ SDBS were added into $100 \mathrm{~mL}$ deionized water, adequately agitated and dispersed with ultrasonic, and then centrifuged at $6000 \mathrm{rpm}$ for $5 \mathrm{~min}$. After that, the centrifuged results were washed with deionized water several times and dried overnight in vacuum to obtain the SDBS-CNFs.

\subsection{Preparation of antistatic superhydrophobic composite coating}

Before spraying, the steel sheets were polished with 800 mesh sandpaper to remove the surface oxide film and then washed with acetone and ethanol for $20 \mathrm{~min}$.

At room temperature, $0.02 \mathrm{~g}$ SDBS-CNFs and $0.07 \mathrm{~g} \mathrm{CeO}_{2}$ particulates were ultrasonically dispersed and stirred for $2 \mathrm{~h}$ in $25 \mathrm{~mL}$ absolute ethyl alcohol. Afterwards, $0.4 \mathrm{~g}$ PPS, $0.3 \mathrm{~g}$ PTFE and $0.1 \mathrm{~g}$ PDMS were successively added into the above homogeneous solution, ultrasonically dispersed and adequately agitated to form a well-dispersed suspension. Subsequently, the prepared dispersed suspension was sprayed onto the substrate surfaces using a $\mathrm{N}_{2}$ gas spray gun. Finally, the antistatic superhydrophobic composite coating (ASC-coating) was obtained by heating the sprayed samples at $330{ }^{\circ} \mathrm{C}$ for $2 \mathrm{~h}$. The schematic diagram of the preparation process of the ASC-coating is shown in Fig. 1.

\subsection{Anti-icing tests}

The anti-icing property was measured by the icing time of droplets on the samples. Deionized water $(35 \mu \mathrm{L})$ was dripped on the surfaces of the specimens and placed into a refrigerator $\left(-10{ }^{\circ} \mathrm{C}\right)$. The relative humidity of the air was approximately $45 \%$, and a digital camera was used

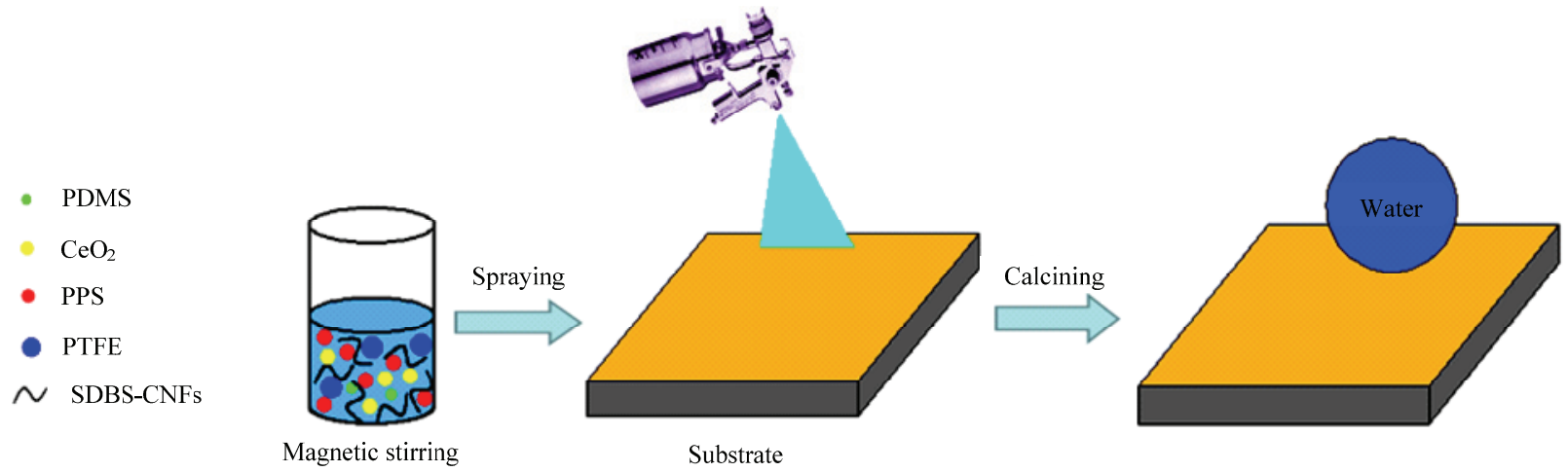

Fig. 1 Schematic diagram of the preparation process of the antistatic superhydrophobic composite coating. 
to observe the changes in the droplet surfaces every $30 \mathrm{~s}$.

\subsection{Thermal stability tests}

The ASC-coating samples were placed into a muffle furnace (KSL-1100X) and heated for $1 \mathrm{~h}$ at 100 ${ }^{\circ} \mathrm{C}, 150{ }^{\circ} \mathrm{C}, 200{ }^{\circ} \mathrm{C}, 250{ }^{\circ} \mathrm{C}, 300{ }^{\circ} \mathrm{C}, 350{ }^{\circ} \mathrm{C}, 400{ }^{\circ} \mathrm{C}$ and $450{ }^{\circ} \mathrm{C}$. After that, the stability was evaluated by measuring the WCAs and SAs of each specimen.

\subsection{Antistatic tests}

A resistivity meter (MCP-T610) with a four-point probe was used to measure the electrical conductivity of samples with different SDBS-CNFs contents. The average values of surface resistance were obtained by measuring five different regions of the sample.

\subsection{Corrosion resistance tests}

The corrosion resistance of the specimens was measured in $3.5 \%$ sodium chloride $(\mathrm{NaCl})$ aqueous solution using a three-electrode electrochemical workstation (CHI660D). The surface area of the working electrode sample is $10 \mathrm{~mm} \times 10 \mathrm{~mm}$. The platinum and saturated calomel electrodes were used as the auxiliary electrode and reference electrode, respectively.

\subsection{Characterization}

The surface morphologies of the particles and coated specimens sputtered with Au before testing were observed by Field Emission Scanning Electron Microscopy (FESEM, JSM-6701F, measured current of $10 \mu \mathrm{A}$, accelerating voltage of $5 \mathrm{kV}$ ). A Fourier Transform Infrared spectrometer (FTIR, Nexus 870) was employed to analyze the chemical compositions in the measuring range of $4000 \mathrm{~cm}^{-1}-400 \mathrm{~cm}^{-1}$. X-ray diffraction (XRD, D8Discover25), with a measuring range of $2 \theta$ from $5^{\circ}$ to $90^{\circ}$, was used to identify the crystal structures of the samples. The WCAs and SAs were measured with $5 \mu \mathrm{L}$ and $8 \mu \mathrm{L}$ deionized water droplets, respectively, on a CA system (JC2000D1CA meter). The average values of WCAs and SAs were obtained by measuring five different regions of the sample. The element distribution and chemical compositon were analyzed by energy-dispersive X-ray spectroscopy (EDS, JSM-5600LV) and X-ray
Photoelectron Spectroscopy (XPS, ESCALAB 250Xi), respectively. Field Emission Transmission Electron Microscopy (FETEM, FEI Tecnai G2 TF20 S-TWIN) was performed to examine the morphology and structure of the particles. A thermogravimetric analyzer (Netzsch STA 449F3) was employed to measure the thermal stability of the coating under a nitrogen atmosphere. The temperature rose from $20{ }^{\circ} \mathrm{C}$ to $800{ }^{\circ} \mathrm{C}$.

\section{Results and discussion}

\subsection{Microstructure characterization of the ASC-coating}

It is well known that the surface microstructure of coatings is extremely vital for hydrophobic property ${ }^{[46]}$. Fig. 2a displays the micromorphology of the as-obtained $\mathrm{CeO}_{2}$ particulates, with diameters ranging from $2 \mu \mathrm{m}$ to $3 \mu \mathrm{m}$, presenting a 3D flowerlike structure, which can provide rough structural support for the ASC-coating. The corresponding XRD pattern is shown in Fig. S1, which indicates the as-prepared $\mathrm{CeO}_{2}$ particulates possess good crystal and cubic fluorite structures (JCPDS Card No. 34-0394). The variations in pristine CNFs before and after modification with SDBS are reflected by FTIR spectroscopy in Fig. S2. By comparison, there are no significant differences between the two curves, indicating that SDBS has no obvious effect on the structure of CNFs. Nevertheless, compared with the CNFs, a new characteristic peak at $1462 \mathrm{~cm}^{-1}$ corresponds to the $\mathrm{C}=\mathrm{C}$ stretching vibration in the benzene ring of SDBS and the absorption peaks at $1130 \mathrm{~cm}^{-1}$ and $675 \mathrm{~cm}^{-1}$ are assigned to the $\mathrm{S}=\mathrm{O}$ stretching vibration of the benzenesulfonic acid group in SDBS $^{[47]}$. The results suggest that the surface of CNFs is successfully coated with SDBS, which is propitious to the uniform distribution of carbon fibers in the ASC-coating.

The SEM image of the $\mathrm{CeO}_{2}$ particulates mixed with SDBS-CNFs $\left(\mathrm{CeO}_{2}\right.$-SDBS-CNFs) is exhibited in Fig. 2b. It can be clearly seen that $\mathrm{CeO}_{2}$ particles and modified CNFs are evenly distributed in the corresponding magnified image (Fig. 2c). By contrast, in Fig. 2d, pristine $\mathrm{CNFs}$ agglomerate with $\mathrm{CeO}_{2}$ particles. The above results show that SDBS can improve the dispersion of CNFs in solution, which is extremely vital to improving the hydrophobicity of the coating. Fig. 2e exhibits the micromorphology of the ASC-coating. From 

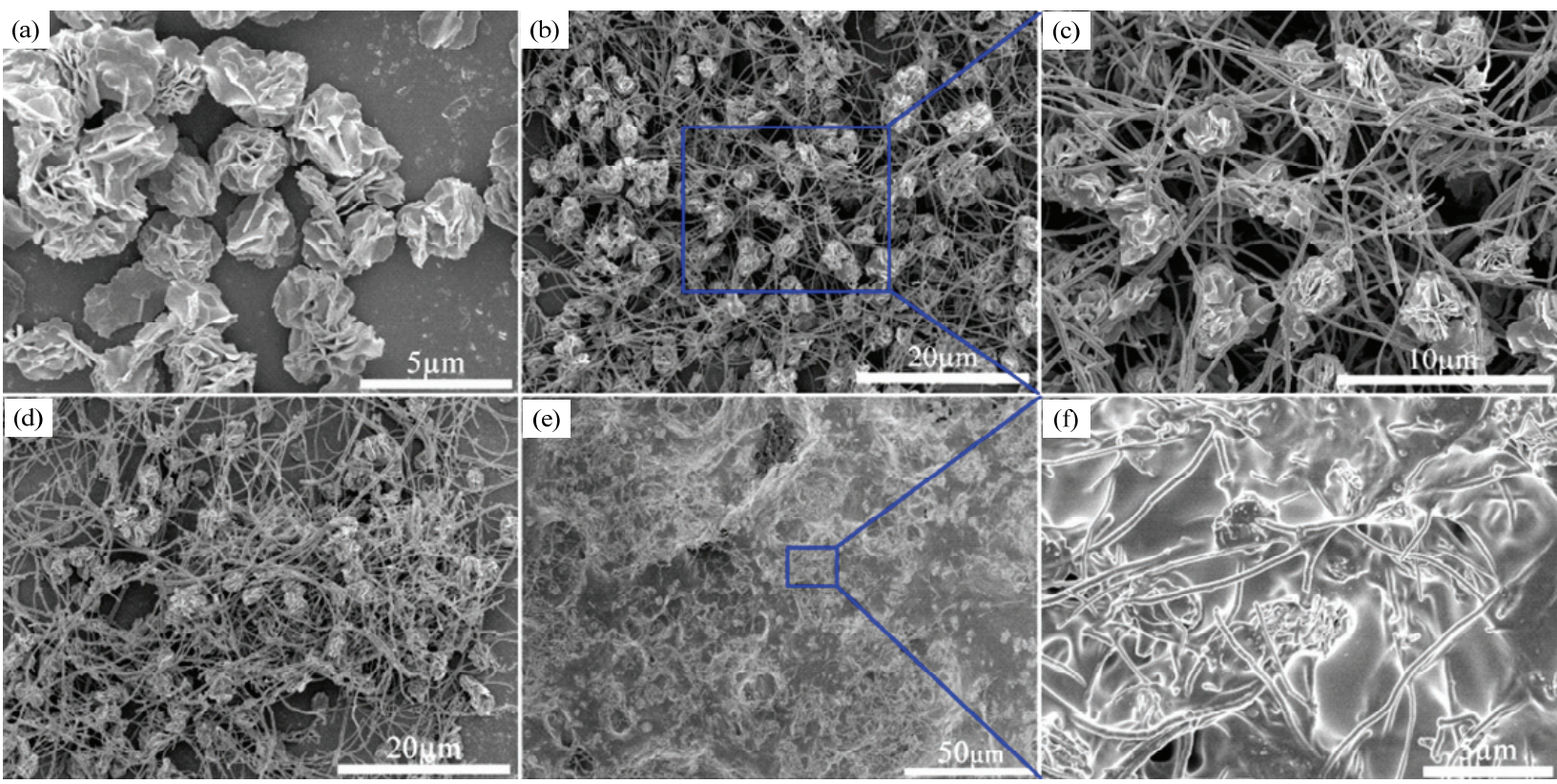

Fig. 2 SEM images of (a) 3D flowerlike $\mathrm{CeO}_{2}$ particulates, (b, c) $\mathrm{CeO}_{2}-\mathrm{SDBS}-\mathrm{CNF}$, (d) $\mathrm{CeO}_{2}-\mathrm{CNF}$, (e, f) ASC-coating.

the magnified image (Fig. 2f), it is clearly visible that SDBS-CNFs, $\mathrm{CeO}_{2}$ particulates and other polymers are evenly crosslinked to form the ASC-coating morphology, which generates a stable micro-nano rough structure for the superhydrophobic coating.

As seen from the Selective Area Electron Diffraction (SAED) pattern in Fig. 3a, the $\mathrm{CeO}_{2}$ particulate exhibits a high polycrystalline structure and all crystal planes match well with the XRD pattern. The clear lattice fringes can be seen from the representative High-Resolution Transmission Electron Microscopy (HRTEM) image (Fig. 3b), which also illustrates the polycrystalline characteristics of the as-prepared particles. Moreover, the lattice fringe spacing is approximately $0.313 \mathrm{~nm}$ (Fig. 3b), which is aligned with those of the (111) planes of ceria particulates in the XRD pattern. The TEM analysis (Fig. 3c) illustrates that CNFs exhibit hollow architectures and 3D flowerlike structures are composed of petals. Furthermore, the magnified TEM image of SDBS-CNFs (Fig. 3d) indicates the SDBS adsorbs well on CNFs, which is consistent with the results in Fig. S2.

From the FTIR spectra of the ASC-coating (Fig. 4a), the peaks at $1468 \mathrm{~cm}^{-1}$ and $1008 \mathrm{~cm}^{-1}$ are assigned to the benzene ring of PPS. The bands at $1089 \mathrm{~cm}^{-1}$ and 478 $\mathrm{cm}^{-1}$ are attributed to the $\mathrm{C}-\mathrm{S}$ bond and $\mathrm{S}-\mathrm{S}$ stretching vibration in PPS. The absorption bands observed at
$1076 \mathrm{~cm}^{-1}, 643 \mathrm{~cm}^{-1}$ and $553 \mathrm{~cm}^{-1}$ are associated with the $\mathrm{C}-\mathrm{C}$ bond, $-\mathrm{CF}^{2-}$ wagging vibration and $-\mathrm{CF}^{2-}$ scissoring vibration for PTFE, respectively. The $-\mathrm{CF}^{2-}$ groups in PTFE provide the essential low surface energy for the ASC-coating. The bands at $1260 \mathrm{~cm}^{-1}$ and $796 \mathrm{~cm}^{-1}$ correspond to the asymmetric bonds of $\mathrm{Si}-\mathrm{O}-\mathrm{C}$ and $\mathrm{Si}-\mathrm{C}$ in PDMS, reducing the surface energy of the ASC-coating. In addition, the strong peaks at $1636 \mathrm{~cm}^{-1}$ should be ascribed to the symmetric stretching vibration of $\mathrm{R}-\mathrm{SO}_{2}-\mathrm{R}$ and the stretching vibration of $\mathrm{S}=\mathrm{O}^{[39]}$, indicating a thermal oxidation crosslinking reaction occurs in PPS. It can further improve the adhesion strength between the Q345 steel sheet and the ASC-coating and play an important role in the formation of the superhydrophobic coating. However, the FTIR characteristic peaks of $\mathrm{CeO}_{2}$ are not observed, which might be explained by the fact that the peak strength is too weak and covered by the other characteristic peaks. According to the XRD pattern of the PPS powder in Fig. $4 \mathrm{~b}$, four diffraction peaks are found at $18.8^{\circ}, 20.6^{\circ}, 25.6^{\circ}$ and $27.4^{\circ}$, which are indexed to the (110), (111/200), (112) and (211) planes of PPS, respectively. After annealing, only the (111/200) plane of PPS is observed in the XRD pattern of the ASC-coating, indicating PPS undergoes a thermal oxidation crosslinking reaction ${ }^{[48]}$. In addition, the diffraction peaks at $18.0^{\circ}$ and $28.4^{\circ}$ are associated with 

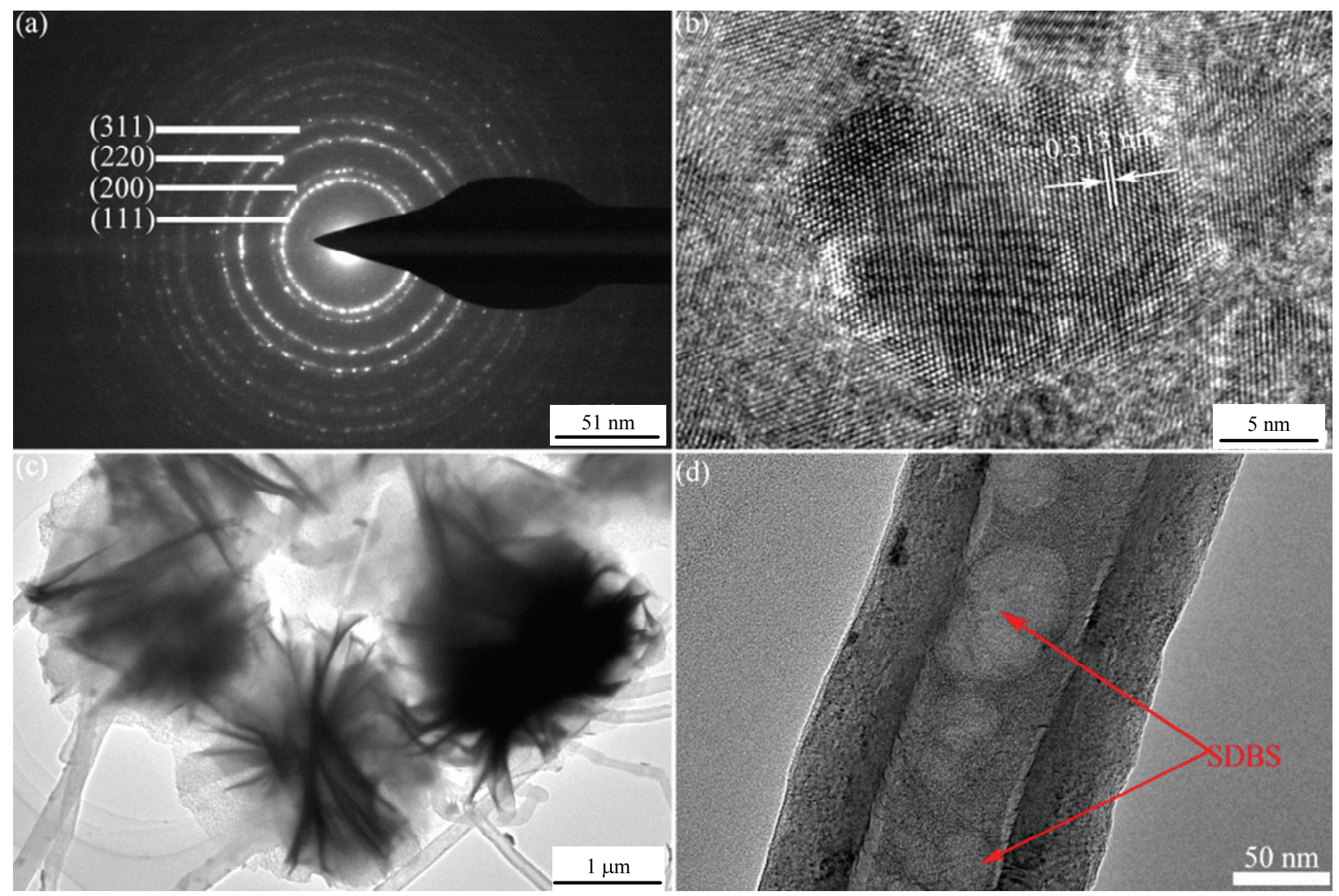

Fig. 3 (a) SAED pattern and (b) HRTEM image of the as-obtained $\mathrm{CeO}_{2}$, TEM images of (c) $\mathrm{CeO}_{2}$-SDBS-CNFs and (d) SDBS-CNFs.

(100) of PTFE and (111) of $\mathrm{CeO}_{2}$, respectively. As presented in Fig. 4c, the F1s at $688.5 \mathrm{eV}$ and S2p at $164.1 \mathrm{eV}$ indicate that PTFE and PPS are dispersed in the superhydrophobic coating. Furthermore, from the EDS spectrum in Fig. 4d, it can be easily seen that the ASC-coating is composed of $\mathrm{C}, \mathrm{O}, \mathrm{F}, \mathrm{Si}, \mathrm{Ce}$ and $\mathrm{S}$. The F, $\mathrm{Si}, \mathrm{Ce}$ and $\mathrm{S}$ peaks are attributed to the existence of PTFE, PDMS, F, $\mathrm{Si}, \mathrm{CeO}_{2}$ and PPS in the ASC-coating, respectively.

\subsection{Wettability}

The hydrophobicity of the coated and uncoated specimens is shown in Figs. 5a-5c. In Fig. 5c, it can be clearly seen that the coated surface (black part) displays excellent water-repellent property. It has a high WCA (166 ${ }^{\circ}$, Fig. 5b) and low SA ( $2^{\circ}$, Fig. 5b). However, compared with the coated surface, the uncoated surface (grey part) in Fig. 5c shows hydrophilicity $\left(65^{\circ}\right.$, Fig. 5a), which indicates the hydrophobic property of the sample surface is obviously improved by the ASC-coating. In addition, the dynamic variations in the water droplet adhesion on the ASC-coating surface are displayed in Fig. S3, which suggests the adhesion force, between the droplet and coating, is very small.

In practical applications, water droplets on the surface of objects tend to be acidic or alkaline ${ }^{[49]}$. Therefore, it is significant to investigate the influence of different $\mathrm{pH}$ values on the surface wettability of samples. The variations in the WCAs and SAs on the surface of specimens at different $\mathrm{pH}$ values ranging from 1 to 14 are exhibited in Fig. 5d. It can be clearly observed from the figure that the WCAs vary steadily with changes in $\mathrm{pH}$ and that the ASC-coating possesses high WCAs $\left(156^{\circ}-166^{\circ}\right)$. With the changes in $\mathrm{pH}$, the SAs fluctuate obviously, but all of them are still less than $10^{\circ}\left(2^{\circ}-\right.$ $5.2^{\circ}$ ). The above analyses indicate that the ASC-coating still displays excellent superhydrophobicity for water droplets with different $\mathrm{pH}$ values.

\subsection{Self-cleaning and anti-fouling properties}

Metal components are easily contaminated in the working environment, which seriously reduces their 

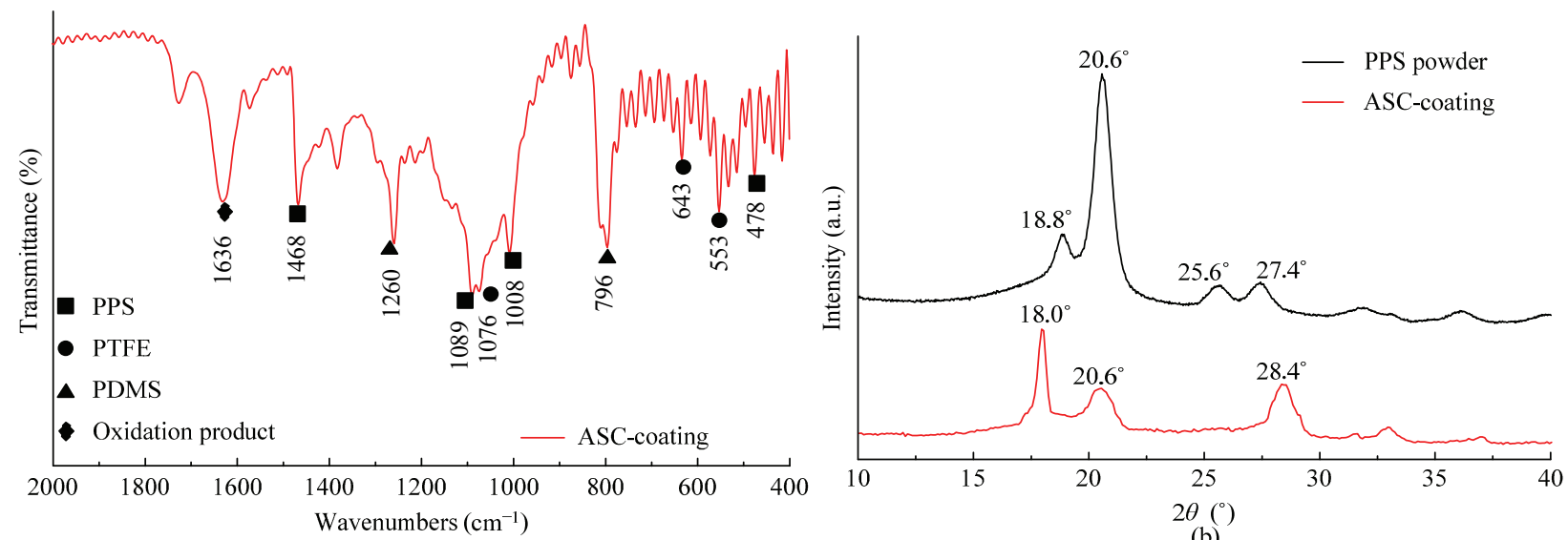

(a)

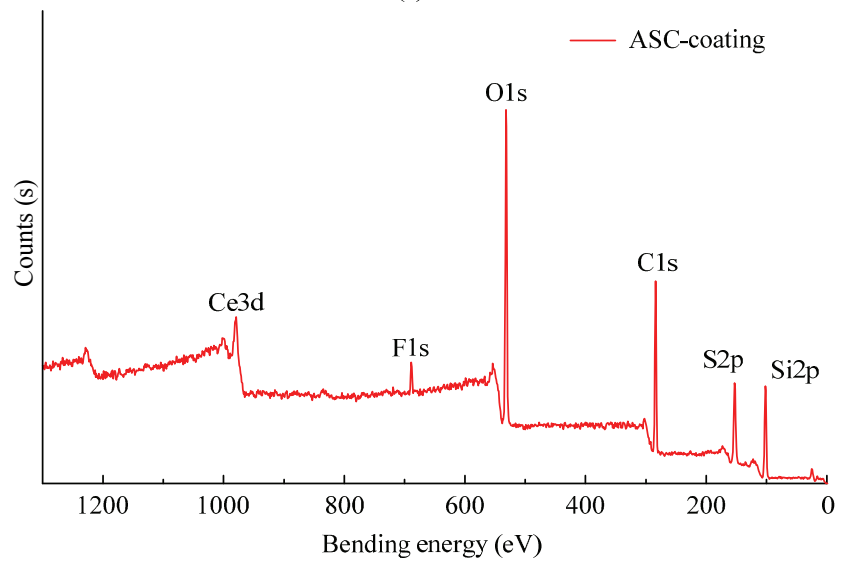

(c)

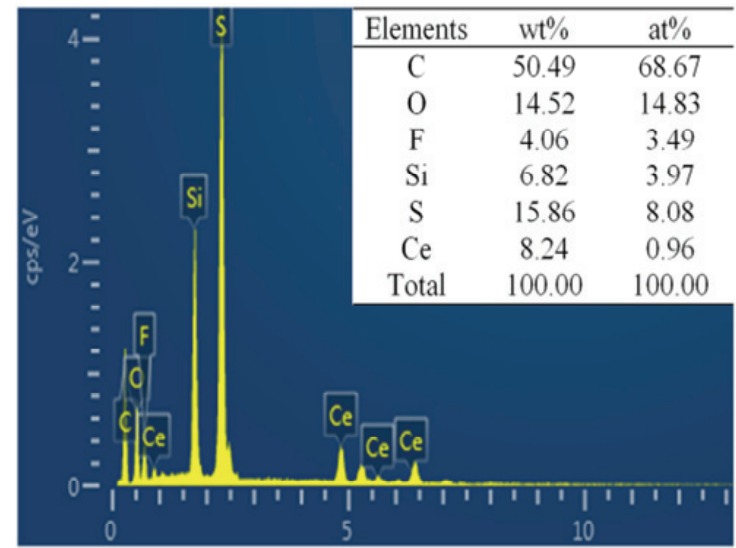

(d)

Fig. 4 (a) FTIR spectra of the ASC-coating, (b) XRD pattern of PPS powder and ASC-coating, (c) XPS spectra and (d) EDS spectrum of ASC-coating.
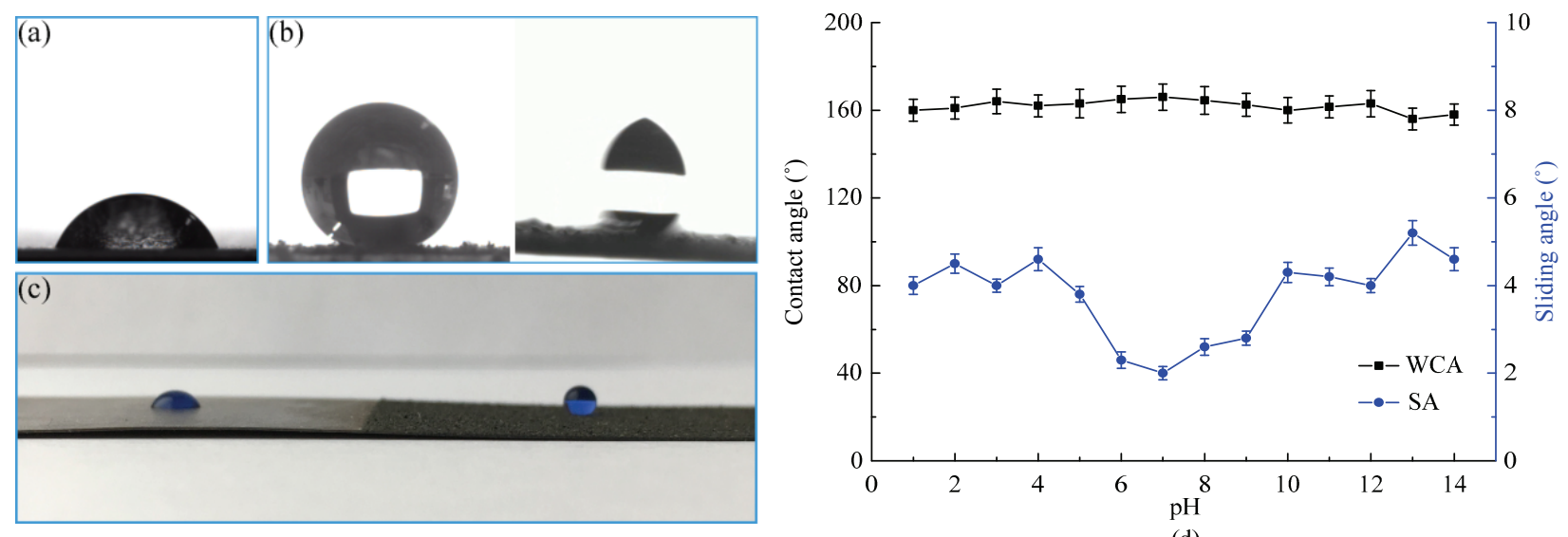

(d)

Fig. 5 (a) Contact angle of Q345, (b) contact and sliding angles of the ASC-coating, (c) photograph of the Q345 (grey part) and ASC-coating (black part), (d) contact and sliding angles of the ASC-coating under different $\mathrm{pH}$ conditions.

service life ${ }^{[50,51]}$. The performances of self-cleaning and anti-fouling can prevent the sample surface from being contaminated in applications. The self-cleaning property of the ASC-coating was investigated using soil from the park. As shown in Figs. 6a and 6b, both coated and uncoated steel sheets are evenly covered with soil. Then the water was slowly dripped onto the contaminated sample surfaces. In Fig. 6a, when water flowed through 
the surface of the untreated steel sheet, a large amount of soil remained on the surface of the sample. In contrast, on the surface of the ASC-coating, water droplets easily rolled off the coating surface and removed the soil from the surface of the sample. As a result, in Fig. 6b, no soil particles were left on the surface of the coated sample, illustrating the excellent self-cleaning performance of the ASC-coating.

The anti-fouling performance of the ASC-coating was evaluated by a muddy water mixed solution, which was made up of water and soil mixed in a certain proportion. After being dripped on the surface of the untreated sample, the muddy water droplets flowed slowly through the surface. Afterwards, a large amount of wet soil adhered to the surface, as shown in Fig. 6c. However, in Fig. 5d, on the ASC-coating surface, the muddy water droplets displayed spherical shape and rapidly rolled down from it. No contaminated particles were left on the surface, which indicated the ASC-coating has outstanding anti-fouling property.

\subsection{Anti-icing property}

The freezing time of water droplets is an important index to evaluate the anti-icing property of the sample surface $^{[52]}$. The superhydrophobic surface possesses an excellent ability to delay ice formation ${ }^{[9]}$. The variations in water droplets with condensation time are shown in Fig. 7. Water droplets were dripped on the coated (in Fig. 7a) and uncoated (in Fig. 7b) surfaces, respectively. With an increase in condensation time, the wetting state of droplets will change. In Fig. 7b, the water droplet on the uncoated surface began to condense into ice at $183 \mathrm{~s}$, while the water droplet on the ASC-coating still showed transparency without ice crystal formation. As time increased, water droplets froze completely at $210 \mathrm{~s}$. Nevertheless, for the coated surface, ice crystals did not appear until $802 \mathrm{~s}$ and white opaque ice droplet appeared at $856 \mathrm{~s}$ (Fig. 7a). The main reasons for the excellent anti-icing performance of the coating surface can be summarized as follows. On the one hand, a small amount of air existed between the droplet and the superhydrophobic surface, which prevented heat transfer and decreased the cooling rate of the water droplets, thereby prolonging the freezing time. On the other hand, the water droplet had a larger contact angle and smaller

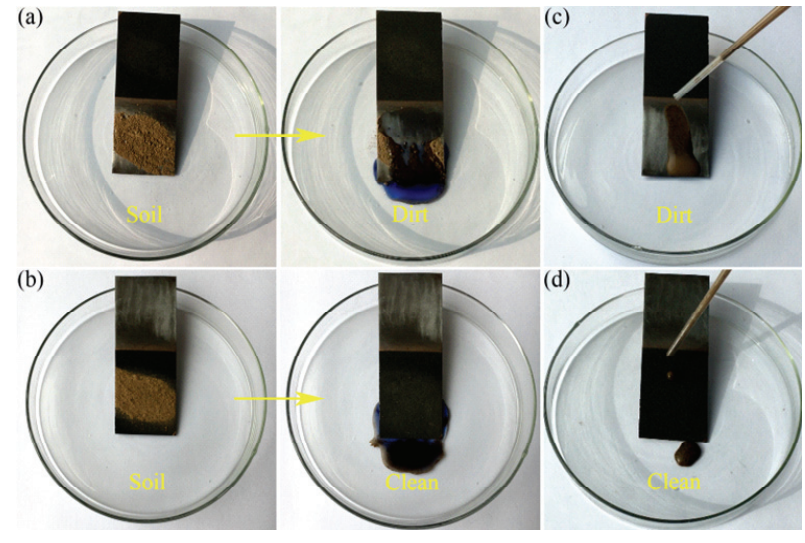

Fig. 6 Self-cleaning property of the Q345 (a) and ASC-coating (b); anti-fouling property of the Q345 (c) and ASC-coating (d).

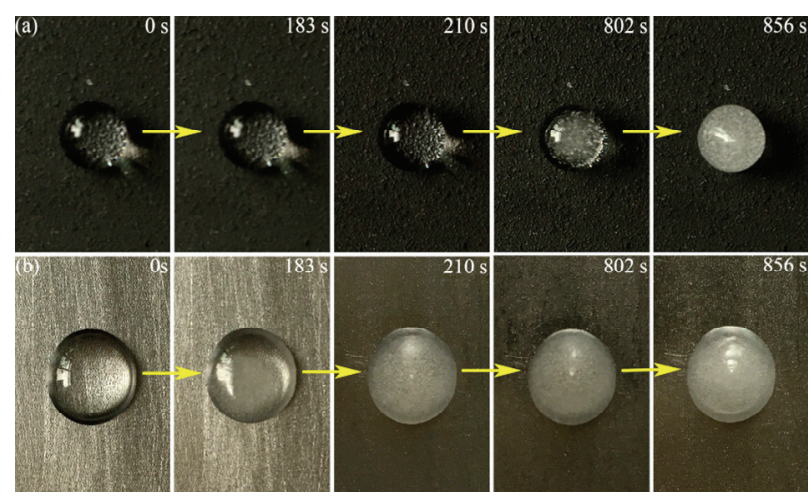

Fig. 7 Variations in droplet icing on the surfaces of (a) coated and (b) uncoated samples at different times.

contact area with the superhydrophobic surface, affecting the speed of heat transfer and slowing down droplet icing $^{[54]}$.

\subsection{Thermal stability}

The thermal stability of superhydrophobic coatings, restricting their further application, is of great significance to their practical application ${ }^{[55]}$. Furthermore, temperature is a significant factor affecting the superhydrophobic properties of coatings ${ }^{[56]}$. The thermogravimetric (TG) and derivative thermogravimetric (DTG) curves of the ASC-coating are displayed in Fig. 8a, and the corresponding thermal parameters are listed in Table S1. For the coating, during thermal decomposition, only one thermogravimetric platform exists in the TG curve. Correspondingly, only one obvious characteristic peak is observed in the DTG curve. As seen from the table, the initial decomposition temperatures of the superhydrophobic coating at 5\% 
$\left(T_{\mathrm{d}} 5 \%\right)$ and $10 \%\left(T_{\mathrm{d}} 10 \%\right)$ weight loss are 496.6 and $519.8{ }^{\circ} \mathrm{C}$, respectively. Moreover, the maximum weight loss rate appears at $553.9{ }^{\circ} \mathrm{C}\left(T_{\max }\right)$ in the DTG curve. Evidently, the above results indicate that the ASC-coating possesses excellent thermal stability which can be explained by the fact that the heat accumulated in the ASC-coating can dissipate quickly due to the excellent thermal conductivity of $\mathrm{CNFs}^{[57]}$. In addition, for the ASC-coating, no significant weight loss is observed below $250{ }^{\circ} \mathrm{C}$. This is mainly attributed to the outstanding stability of the ASC-coating compositions at $250{ }^{\circ} \mathrm{C}$.

Fig. 8 b shows the superhydrophobic variations in the ASC-coating surface with heating temperature. With increasing heating temperature, the WCAs decrease and SAs increase, but the ASC-coating surface still displays superhydrophobicity below $350{ }^{\circ} \mathrm{C}$ with high WCAs $\left(151^{\circ}-162^{\circ}\right)$ and low SAs $\left(3^{\circ}-8^{\circ}\right)$, illustrating the great superhydrophobic thermal stability of the coating surface. However, the coating, with $141^{\circ} \mathrm{WCA}$ and $15^{\circ}$ SA, loses its superhydrophobicity at $400{ }^{\circ} \mathrm{C}$ because of the thermal decomposition of PTFE and PDMS. When the heating temperature is further increased to $500{ }^{\circ} \mathrm{C}$, the WCA of the ASC-coating surface is $0^{\circ}$, which is ascribed to the thermal decomposition of the coating at $496{ }^{\circ} \mathrm{C}$.

\subsection{Antistatic analysis}

Surface Resistivity (SR) can be applied to measure the antistatic properties of coatings ${ }^{[58]}$. Table S2 shows the surface resistivity and logarithmic values of the superhydrophobic coating with different carbon contents ( $g$ and $w t \%$ ). With the increase in SDBS-CNFs content (g), the variations in the logarithmic values and WCAs of the superhydrophobic coating are displayed in Fig. 9a. From Fig. 9b, it is found that there are not enough carbon fibers to form continuous conductive channels in the coating, which results in the SR $\left(3.96 \times 10^{8} \Omega\right)$ being very high at $0.005 \mathrm{~g}$ SDBS-CNFs. With the SDBS-CNFs content increasing to $0.010 \mathrm{~g}$, the surface resistivity decreases sharply to $2.82 \times 10^{5} \Omega$, achieving an antistatic standard, which is ascribed to the formation of continuous conductive paths (as shown in Fig. 9c). This result indicates the antistatic property of the ASC-coating increases with increasing SDBS-CNFs content. As the SDBS-CNFs content continuously increases, the SR of the ASC-coating varies slowly, which indicates the coating has an electrical percolation effect. Furthermore, the electrical percolation threshold $(\Phi)$ is approximately $1.12 \mathrm{wt} \%$, corresponding to an SDBS-CNFs content of $0.010 \mathrm{~g}$. Once the SDBS-CNFs content exceeds $\Phi$, the antistatic property of the coating is greatly improved. As the SDBS-CNFs content increases from $0.015 \mathrm{~g}$ to $0.030 \mathrm{~g}$, the corresponding SR, falling from $3.76 \times 10^{4} \Omega$ to $3.47 \times 10^{3} \Omega$, decreases increasingly slowly. Moreover, with further SDBS-CNFs addition, no obvious variations in the SR are found in the curve. Correspondingly, in Fig. 9d, the SDBS-CNFs distribute on the coating surface to form continuous conductive channels, which is not

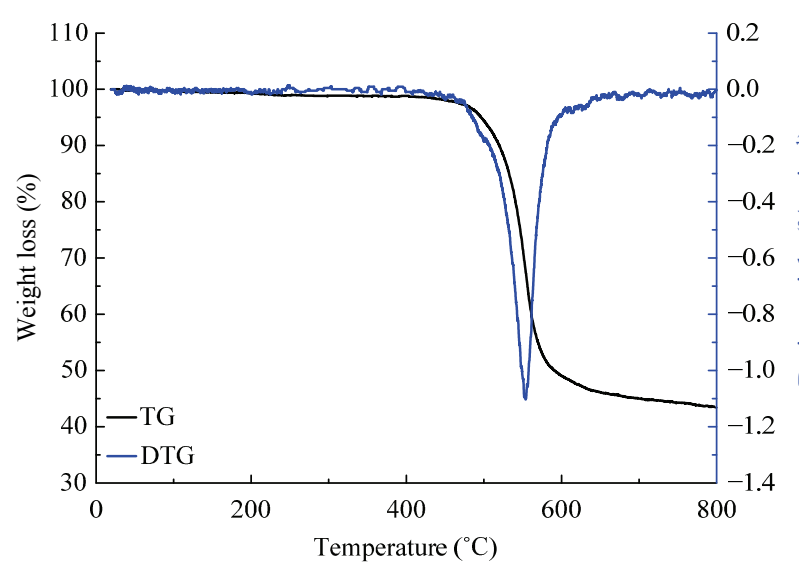

(a)

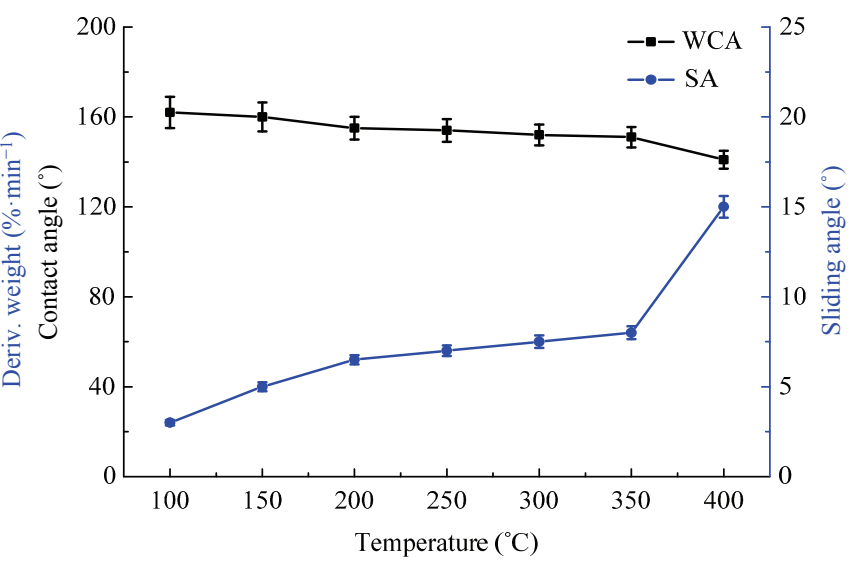

(b)

Fig. 8 (a) TG and DTG curves of the ASC-coating, (b) variations in the contact and sliding angles of the ASC-coating at different temperatures. 

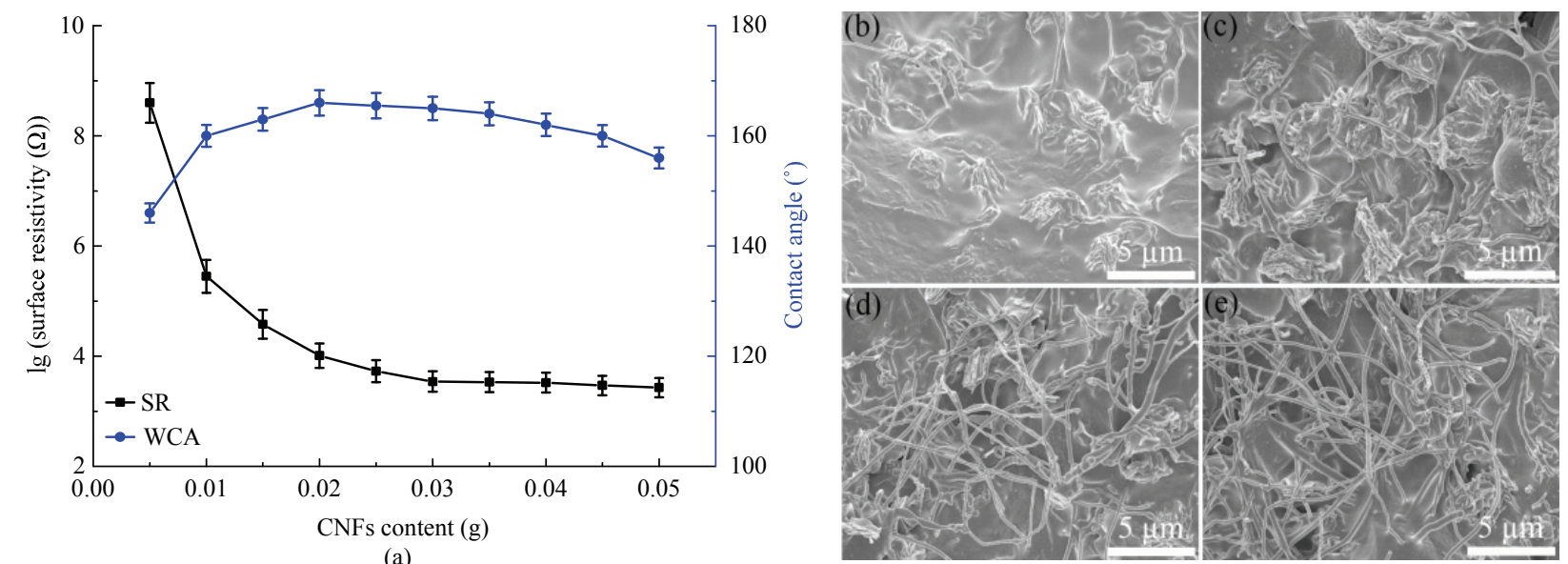

Fig. 9 (a) Variations in the surface resistivity and contact angle of the ASC-coating at different CNFs contents; SEM images of the ASC-coating with (b) $0.005 \mathrm{~g}$, (c) $0.01 \mathrm{~g}$, (d) $0.03 \mathrm{~g}$ and (e) $0.04 \mathrm{~g} \mathrm{CNFs}$.

significantly different from the morphology shown in Fig. 9e. The above results can be explained by the growth process of the percolation group. The percolation ratio is the ratio of the content of SDBS-CNFs in the continuous conductive channels and total SDBS-CNFs ${ }^{[59]}$. At the initial stage, a small addition in SDBS-CNFs content will significantly increase the percolation ratio, causing a rapid decrease in the SR. Furthermore, near $\Phi$, the growth rate of the percolation group is extremely rapid. Nevertheless, when the SDBS-CNFs content exceeds the critical value $\Phi$, the growth rate of the percolation group begins to decrease, and the corresponding SR of the ASC-coating decreases increasingly slowly with increasing SDBS-CNFs content. As the SDBS-CNFs content exceeds $0.030 \mathrm{~g}$, the percolation ratio is close to $100 \%$, resulting in the growth rate of the percolation group no longer varying significantly with increasing SDBS-CNFs content. As a result, the SR tends to be stable.

It can also be seen from Fig. 9a that the hydrophobicity of the coating is related to the SDBS-CNFs content. Compared with the SR, the WCA $\left(146^{\circ}\right)$ is less than $150^{\circ}$ at low SDBS-CNFs contents (< $0.005 \mathrm{~g}$ ), which is attributed to insufficient SDBS-CNFs to construct superhydrophobic rough structures. After that, as the SDBS-CNFs content increases, the hydrophobicity of the ASC-coating increases gradually due to the improvement of the surface hydrophobic rough structure. In particular, by increasing the SDBS-CNFs content to $0.020 \mathrm{~g}$, the ASC-coating possesses the best superhydrophobicity, with a WCA of $166^{\circ}$. The corresponding SR is $8.70 \times 10^{3} \Omega$, illustrating the coating has outstanding antistatic property. However, with further SDBS-CNFs addition $(>0.020 \mathrm{~g}$ ), the dispersion of SDBS-CNFs in the superhydrophobic coatings decreases, causing a decrease in hydrophobicity. As a result, the WCA of the coating decreases continuously. From the above analysis results, it can be seen that the optimum SDBS-CNFs content is $0.020 \mathrm{~g}$, at which the ASC-coating possesses both optimal hydrophobicity and excellent antistatic property.

\subsection{Corrosion resistance}

Corrosion resistance is an important index to measure the service performance of iron and steel ${ }^{[3,5]}$. Superhydrophobic coatings can improve the corrosion resistance of matrix samples. The Tafel plots of the uncoated and different coated samples in $3.5 \% \mathrm{NaCl}$ aqueous solution are characterized in Fig. 10a. The corrosion resistance of the coating is evaluated through the corrosion potential $\left(E_{\text {corr }}\right)$ and corrosion current $\left(I_{\text {corr }}\right)$ obtained using Tafel extrapolation (as shown in Table S3). In general, higher $E_{\text {corr }}$ and smaller $I_{\text {corr }}$ indicate lower rates of thermodynamic corrosion and dynamic corrosion, respectively ${ }^{[60]}$. Compared with the Tafel plot of the bare Q345 steel sheet $\left(E_{\text {corr }}=-0.603 \mathrm{~V}, I_{\text {corr }}=\right.$ $3.166 \times 10^{-5} \mathrm{~A}$ ), it can be clearly seen that the corrosion resistance performance was drastically improved on the ASC-coating surface as a result of the higher corrosion potential $(-0.378 \mathrm{~V})$ and lower corrosion current 


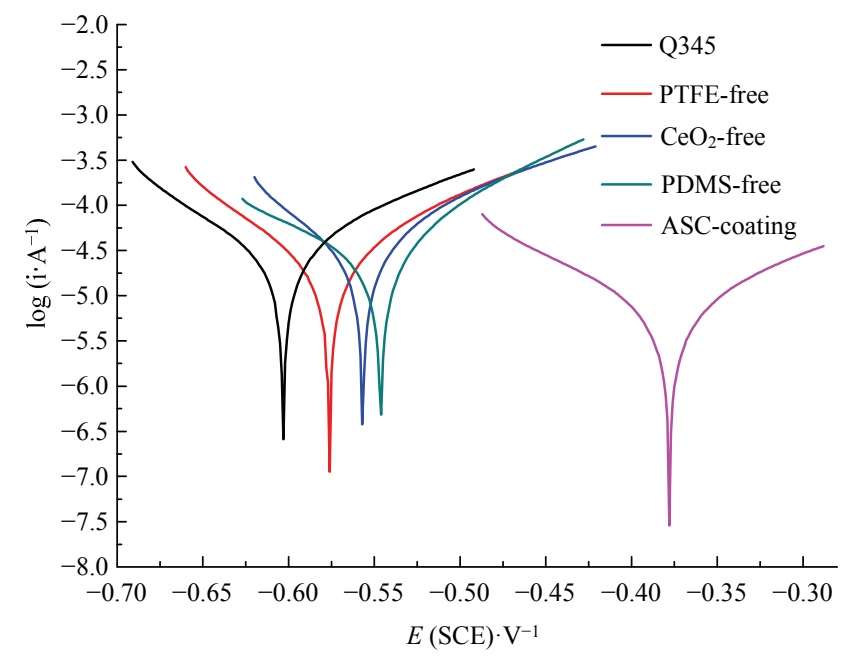

(a)

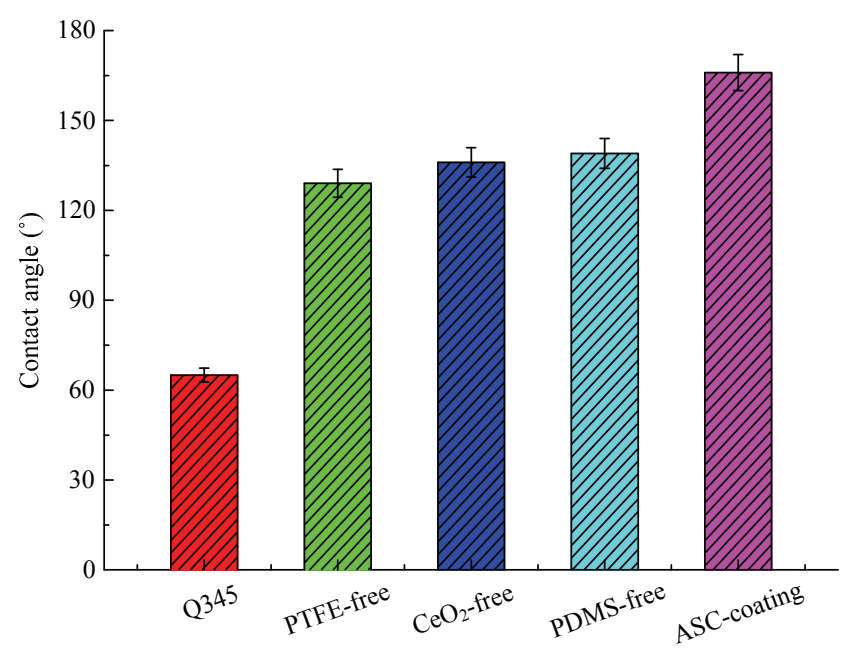

(b)

Fig. 10 (a) Tafel plots of the bare and differently coated Q345 steel sheets in a 3.5\% $\mathrm{NaCl}$ aqueous solution. The differently coated Q345 steel sheets include the samples of PTFE-free coating, $\mathrm{CeO}_{2}$-free coating, PDMS-free coating and ASC-coating. (b) Contact angle of the bare and differently coated Q345 steel sheets including the PTFE-free coating, $\mathrm{CeO}_{2}$-free coating, PDMS-free coating and ASC-coating.

$\left(6.439 \times 10^{-6}\right.$ A). According to a previous study ${ }^{[53]}$, the improvement of corrosion resistance might be attributed to the existence of air bubbles in the micro-nano architectures of the coating. Owing to the low surface energy of the superhydrophobic coating, the corrosive solution molecules are prevented from penetrating into the micro-nano architectures of the coating, bringing about the better anticorrosion property.

However, in general, the barrier effect provided by coatings can also enhance the corrosion resistance of the matrix ${ }^{[61]}$. Therefore, it is uncertain whether the good corrosion performance is determined by the shielding effect or the superhydrophobic ability. Based on the above indeterminacy, the anticorrosion properties of three other contrast coated samples, namely, the PTFE-free coating sample (PTFE-free), $\mathrm{CeO}_{2}$-free coating sample $\left(\mathrm{CeO}_{2}\right.$-free) and PDMS-free coating sample (PDMS-free), were researched under the same conditions. As seen from the Tafel plots of three other coated samples, the corrosion potentials of PTFE-free, $\mathrm{CeO}_{2}$-free and PDMS-free samples are $-0.576 \mathrm{~V}$, $-0.557 \mathrm{~V}$ and $-0.546 \mathrm{~V}$, respectively, and the corresponding corrosion currents are $2.581 \times 10^{-5} \mathrm{~A}$, $1.833 \times 10^{-5} \mathrm{~A}$ and $1.502 \times 10^{-5} \mathrm{~A}$, illustrating the anticorrosion property of the substrate is enhanced by the barrier effect of the three coatings. Nevertheless, the anticorrosion performance of the ASC-coating is obviously better than that of the other three coatings, which is closely related to the hydrophobic properties of several coatings. As presented in Fig. 10b, the WCAs (increasing from $129^{\circ}$ to $166^{\circ}$ ) of the four coatings increase in the order of PTFE-free, $\mathrm{CeO}_{2}$-free, PDMS-free, and ASC-coating, which is consistent with the variation tendency of their anticorrosive properties. It can be explained that the contact area between the corrosive solution and coating surface with poor hydrophobicity increases, and the corrosive liquid molecules can easily penetrate into the micro-nano architectures of the coating surface. Specifically, for the superhydrophobic coating, the anticorrosion ability of the surface increases dramatically due to the air protection shield on the superhydrophobic surface ${ }^{[62]}$. Consequently, it can be proven that the improvement of corrosion resistance on the ASC-coating surface depends mainly on superhydrophobic behavior rather than on the coating barrier.

\section{Conclusion}

In summary, a facile and modifier-free approach was applied to fabricate a PPS-based antistatic superhydrophobic coating. The as-obtained functional coating exhibits remarkable superhydrophobicity, a high water contact angle $\left(166^{\circ}\right)$ and a low sliding angle $\left(2^{\circ}\right)$. It has been shown that the coating, with outstanding water repellency stability under different $\mathrm{pH}$ conditions, possesses excellent self-cleaning, anti-fouling and 
anti-icing properties. Due to the excellent thermal conductivity of CNFs, the coating still maintains its superhydrophobicity after calcination at $350{ }^{\circ} \mathrm{C}$ for $1 \mathrm{~h}$. In addition, the surface resistivity and superhydrophobicity of the prepared coating are related to the SDBS-CNFs content. When the SDBS-CNFs content is $0.020 \mathrm{~g}$, the antistatic superhydrophobic coating possesses both optimal hydrophobicity and excellent antistatic property. Furthermore, the superhydrophobic coating displays excellent corrosion resistance in a $3.5 \% \mathrm{NaCl}$ aqueous solution. This approach with a facile and inexpensive preparation process may make possible true industrial applications. In addition to the common application areas, this kind of functional coating can be used in some special fields such as coal mining, aviation, defense and petrochemical applications.

\section{Acknowledgment}

This work is supported by the National Nature Science Foundation of China (Grant Nos. 51735013, 51675513 and 51875564).

* All supplementary materials are available at https://doi.org/10.1007/s42235-020-0034-3.

Open Access This article is licensed under a Creative Commons Attribution 4.0 International License, which permits use, sharing, adaptation, distribution and reproduction in any medium or format, as long as you give appropriate credit to the original author(s) and the source, provide a link to the Creative Commons licence, and indicate if changes were made.

The images or other third party material in this article are included in the article's Creative Commons licence, unless indicated otherwise in a credit line to the material. If material is not included in the article's Creative Commons licence and your intended use is not permitted by statutory regulation or exceeds the permitted use, you will need to obtain permission directly from the copyright holder.

To view a copy of this licence, visit $\mathrm{http}: / /$ creativecommons.org/licenses/by/4.0/.

\section{References}

[1] Masood M T, Heredia-Guerrero J A, Ceseracciu L, Palazon F, Athanassiou A, Bayer I S. Superhydrophobic high impact polystyrene (HIPS) nanocomposites with wear abrasion resistance. Chemical Engineering Journal, 2017, 322, $10-21$.

[2] Ma Q, Tong Z, Wang W, Dong G N. Fabricating robust and repairable superhydrophobic surface on carbon steel by nanosecond laser texturing for corrosion protection. Applied Surface Science, 2018, 455, 748-757.

[3] Qian H C, Xu D K, Du C W, Zhang D W, Li X G, Huang L Y, Deng L P, Tu Y C, Mol J M, Terryn H A. Dual-action smart coatings with a self-healing superhydrophobic surface and anti-corrosion properties. Journal of Materials Chemistry A, 2017, 5, 2355-2364.

[4] Fan Y, He Y, Luo P Y, Chen X, Liu B. A facile electrodeposition process to fabricate corrosion-resistant superhydrophobic surface on carbon steel. Applied Surface Science, 2016, 368, 435-442.

[5] Liu Q, Chen D X, Kang Z X. One-step electrodeposition process to fabricate corrosion-resistant superhydrophobic surface on magnesium alloy. ACS Applied Materials \& Interfaces, 2015, 7, 1859-1867.

[6] Cao C Y, Ge M Z, Huang J Y, Li S H, Deng S, Zhang S N, Chen Z, Zhang K Q, Al-Deyab S S, Lai Y K. Robust fluorine-free superhydrophobic PDMS-ormosil@fabrics for highly effective self-cleaning and efficient oil-water separation. Journal of Materials Chemistry A, 2016, 4, 12179-12187.

[7] Liu H, Huang J Y, Chen Z, Chen G Q, Zhang K Q, Al-Deyab $\mathrm{S}$ S, Lai Y K. Robust translucent superhydrophobic PDMS/PMMA film by facile one-step spray for self-cleaning and efficient emulsion separation. Chemical Engineering Journal, 2017, 330, 26-35.

[8] Wang L, Gong Q H, Zhan S H, Jiang L, Zheng Y M. Robust anti-icing performance of a flexible superhydrophobic surface. Advanced Materials, 2016, 28, 7729-7735.

[9] Zhan X L, Yan Y D, Zhang Q H, Chen F Q. A novel superhydrophobic hybrid nanocomposite material prepared by surface-initiated AGET ATRP and its anti-icing properties. Journal of Materials Chemistry A, 2014, 2, 9390-9399.

[10] Liu W L, Chen H F, Shen Y Z, Wu Z W. Facilely fabricating superhydrophobic resin-based coatings with lower water freezing temperature and ice adhesion for anti-icing application. Journal of Bionic Engineering, 2019, 16, 794-805.

[11] Hu H B, Wen J, Bao L Y, Jia L B, Song D, Song B W, Pan G, Scaraggi M, Dini D, Xue Q J, Zhou F. Significant and stable drag reduction with air rings confined by alternated 
superhydrophobic and hydrophilic strips. Science Advances, 2017, 3, 1-9.

[12] Cheng M J, Song M M, Dong H Y, Shi F. Surface adhesive forces: a metric describing the drag-reducing effects of superhydrophobic coatings. Small, 2015, 11, 1665-1671.

[13] Su X J, Li H Q, Lai X J, Zhang L, Wang J, Liao X F, Zeng X R. Vapor-liquid sol-gel approach to fabricating highly durable and robust superhydrophobic polydimethylsiloxane@silica surface on polyester textile for oil-water separation. ACS Applied Materials \& Interfaces, 2017, 9, 28089-28099.

[14] Latthe S S, Terashima C, Nakata K, Sakai M, Fujishima A. Development of sol-gel processed semi-transparent and self-cleaning superhydrophobic coatings. Journal of Materials Chemistry A, 2014, 2, 5548-5553.

[15] Zhang X X, Cai S, You D, Yan L H, Lv H B, Yuan X D, Jiang B. Template-free sol-gel preparation of superhydrophobic ORMOSIL films for double-wavelength broadband antireflective coatings. Advanced Functional Materials, 2013, 23, 4361-4365.

[16] Peng P P, Ke Q P, Zhou G, Tang T D. Fabrication of microcavity-array superhydrophobic surfaces using an improved template method. Journal of Colloid and Interface Science, 2013, 395, 326-328.

[17] Gong D W, Long J Y, Jiang D F, Fan P X, Zhang H J, Li L, Zhong M L. Robust and stable transparent superhydrophobic polydimethylsiloxane films by duplicating via a femtosecond laser-ablated template. ACS Applied Materials \& Interfaces, 2016, 8, 17511-17518.

[18] Timonen J V, Latikka M, Leibler L, Ras R H, Ikkala O. Switchable static and dynamic self-assembly of magnetic droplets on superhydrophobic surfaces. Science, 2013, 341, 253-257.

[19] Jiang H J, Zhang L, Chen J, Liu M H. Hierarchical self-assembly of a porphyrin into chiral macroscopic flowers with superhydrophobic and enantioselective property. ACS Nano, 2017, 11, 12453-12460.

[20] Xue C H, Li Y R, Zhang P, Ma J Z, Jia S T. Washable and wear-resistant superhydrophobic surfaces with self-cleaning property by chemical etching of fibers and hydrophobization. ACS Applied Materials \& Interfaces, 2014, 6, 10153-10161.

[21] Huang Y, Sarkar D K, Chen X G. Superhydrophobic aluminum alloy surfaces prepared by chemical etching process and their corrosion resistance properties. Applied Surface Science, 2015, 356, 1012-1024.

[22] Rezayi T, Entezari M H. Toward a durable superhydrophobic aluminum surface by etching and $\mathrm{ZnO}$ nanoparticle deposition. Journal of Colloid and Interface Science, 2016, 463, 37-45.

[23] Deng X, Mammen L, Butt H J, Vollmer D. Candle soot as a template for a transparent robust superamphiphobic coating. Science, 2012, 335, 67-70.

[24] Zhang Y, Ge D T, Yang S. Spray-coating of superhydrophobic aluminum alloys with enhanced mechanical robustness. Journal of Colloid and Interface Science, 2014, 423, 101-107.

[25] Wang T, Ge H Y, Zhang K L. A novel core-shell silica@graphene straticulate structured antistatic anticorrosion composite coating. Journal of Alloys and Compounds, 2018, 745, 705-715.

[26] Song H P, Liu J Q, Xue F B, Cheng F Q. The application of ultra-fine fly ash in the seal coating for the wall of underground coal mine. Advanced Powder Technology, 2016, 27, 1645-1650.

[27] Samaha M A, Tafreshi H V, Gad-El-Hak M. Influence of flow on longevity of superhydrophobic coatings. Langmuir, 2012, 28, 9759-9766.

[28] Yin L, Wang Y Y, Ding J F, Wang Q J, Chen Q M. Water condensation on superhydrophobic aluminum surfaces with different low-surface-energy coatings. Applied Surface Science, 2012, 258, 4063-4068.

[29] Galletti A M, Antonetti C, Marracci M, Piccinelli F, Tellini B. Novel microwave-synthesis of $\mathrm{Cu}$ nanoparticles in the absence of any stabilizing agent and their antibacterial and antistatic applications. Applied Surface Science, 2013, 280, 610-618.

[30] Balram A, Santhanagopalan S, Hao B Y, Yap Y K, Meng D D. Electrophoretically-deposited metal-decorated CNT nanoforests with high thermal/electric conductivity and wettability tunable from hydrophilic to superhydrophobic. Advanced Functional Materials, 2016, 26, 2571-2579.

[31] Zhang Y Q, Yang S, Wang S L, Liu H K, Li L, Dou S X, Liu $\mathrm{X}$. Engineering high-performance $\mathrm{MoO}_{2}$-based nanomaterials with supercapacity and superhydrophobicity by tuning the raw materials source. Small, 2018, 14, 1-9.

[32] Das A, Hayvaci H T, Tiwari M K, Bayer I S, Erricolo D, Megaridis C M. Superhydrophobic and conductive carbon nanofiber/PTFE composite coatings for EMI shielding. Journal of Colloid and Interface Science, 2011, 353, 311-315.

[33] Wang P, Sun B, Liang Y, Han H L, Fan X L, Wang W L, Yang Z. A stretchable and super-robust graphene superhydrophobic composite for electromechanical sensor application. Journal of Materials Chemistry A, 2018, 6, 
10404-10410.

[34] Yao W H, Bae K J, Jung M Y, Cho Y R. Transparent, conductive, and superhydrophobic nanocomposite coatings on polymer substrate. Journal of Colloid and Interface Science, 2017, 506, 429-436.

[35] Mates J E, Bayer I S, Palumbo J M, Carroll P J, Megaridis C M. Extremely stretchable and conductive water-repellent coatings for low-cost ultra-flexible electronics. Nature Communications, 2015, 6, 1-8.

[36] He Y T, Wang L X, Jia D Z. Coal/PAN interconnected carbon nanofibers with excellent energy storage performance and electrical conductivity. Electrochimica Acta, 2016, 194, 239-245.

[37] Azimi G, Dhiman R, Kwon H M, Paxson A T, Varanasi K K. Hydrophobicity of rare-earth oxide ceramics. Nature Materials, 2013, 12, 315-320.

[38] Yu X F, Liu J W, Cong H P, Xue L, Arshad M N, Albar H A, Sobahi T R, Gao Q, Yu S H. Template- and surfactant-free synthesis of ultrathin $\mathrm{CeO}_{2}$ nanowires in a mixed solvent and their superior adsorption capability for water treatment. Chemical Science, 2015, 6, 2511-2515.

[39] Qi H M, Zhang L G, Zhang G, Wang T M, Wang Q H. Comparative study of tribochemistry of ultrahigh molecular weight polyethylene, polyphenylene sulfide and polyetherimide in tribo-composites. Journal of Colloid and Interface Science, 2018, 514, 615-624.

[40] Luo D, Chen M, Xu J, Yin X Z, Wu J, Chen S H, Wang L X, Wang H. Polyphenylene sulfide nonwoven-based composite separator with superior heat-resistance and flame retardancy for high power lithium ion battery. Composites Science \& Technology, 2018, 157, 119-125.

[41] Feng S S, Zhong Z X, Zhang F, Wang Y, Xing W H. Amphiphobic polytetrafluoroethylene membranes for efficient organic aerosol removal. ACS Applied Materials \& Interfaces, 2016, 8, 8773-8781.

[42] Shi L T, Hu J, Lin X D, Fang L, Wu F, Xie J, Meng F M. A robust superhydrophobic PPS-PTFE/SiO ${ }_{2}$ composite coating on AZ31 Mg alloy with excellent wear and corrosion resistance properties. Journal of Alloys and Compounds, 2017, 721, 157-163.

[43] Wang H Y, Yan L, Gao D, Liu D J, Wang C, Sun L Y, Zhu Y J. Tribological properties of superamphiphobic PPS/PTFE composite coating in the oilfield produced water. Wear, 2014, 319, 62-68.

[44] Wang H Y, Zhao J Y, Zhu Y Z, Meng Y, Zhu Y J. The fabrication, nano/micro-structure, heat- and wear-resistance of the superhydrophobic PPS/PTFE composite coatings.
Journal of Colloid and Interface Science, 2013, 402, 253-258.

[45] Zhong L S, Hu J S, Cao A M, Liu Q, Song W G, Wan L J. 3D flowerlike ceria micro/nanocomposite structure and its application for water treatment and $\mathrm{CO}$ removal. Chemistry of Materials, 2007, 19, 1648-1655.

[46] Lv T, Cheng Z J, Zhang E S, Kang H J, Liu Y Y, Jiang L. Self-restoration of superhydrophobicity on shape memory polymer arrays with both crushed microstructure and damaged surface chemistry. Small, 2016, 13, 1-8.

[47] Liu J, Wang D P, Gao L X, Zhang D Q. Synergism between cerium nitrate and sodium dodecylbenzenesulfonate on corrosion of AA5052 aluminium alloy in 3 wt.\% $\mathrm{NaCl}$ solution. Applied Surface Science, 2016, 389, 369-377.

[48] Xing J, Ni Q Q, Deng B Y, Liu Q S. Morphology and properties of polyphenylene sulfide (PPS)/polyvinylidene fluoride (PVDF) polymer alloys by melt blending. Composites Science \& Technology, 2016, 134, 184-190.

[49] $\mathrm{Hu} \mathrm{H} \mathrm{B,} \mathrm{Gao} \mathrm{L,} \mathrm{Chen} \mathrm{C} \mathrm{L,} \mathrm{Chent} \mathrm{Q} \mathrm{W.} \mathrm{Low-cost,}$ acid/alkaline-resistant, and fluorine-free superhydrophobic fabric coating from onionlike carbon microspheres converted from waste polyethylene terephthalate environ. Environmental Science \& Technology, 2014, 48, 2928-2933.

[50] Yin L, Zhang H F, Li Y Y, Wang Y, Zhang R M, Chen W P, Liu X W. Fabrication of biomimetic superhydrophobic steel surface under an oxygen rich environment. Applied Surface Science, 2016, 380, 40-46.

[51] Gao X Y, Guo Z G. Mechanical stability, corrosion resistance of superhydrophobic steel and Rrpairable durability of its slippery surface. Journal of Colloid and Interface Science, 2018, 512, 239-248.

[52] Liao R J, Zuo Z P, Guo C, Yuan Y, Zhuang A Y. Fabrication of superhydrophobic surface on aluminum by continuous chemical etching and its anti-icing property. Applied Surface Science, 2014, 317, 701-709.

[53] Wang N, Xiong D S, Deng Y L, Shi Y, Wang K. Mechanically robust superhydrophobic steel surface with anti-icing, UV-durability, and corrosion resistance properties. ACS Applied Materials \& Interfaces, 2015, 7, 6260-6272.

[54] Zou X S, Tao C Y, Yang K, Yang F, Lv H B, Yan L H, Yan H W, Li Y, Xie Y Y, Yuan X D, Zhang L. Rational design and fabrication of highly transparent, flexible, and thermally stable superhydrophobic coatings from raspberry-like hollow silica nanoparticles. Applied Surface Science, 2018, 440, 700-711. 
[55] Xiao Z, Zhang L Q, Zhang W W, Yu X Q, Zhang Y F. Thermal stability of typical superhydrophobic surfaces. Journal of Bionic Engineering, 2018, 15, 1025-1034.

[56] Gong G M, Gao K, Wu J T, Sun N, Zhou C, Zhao Y, Jiang L. A highly durable silica/polyimide superhydrophobic nanocomposite film with excellent thermal stability and abrasion-resistant performance. Journal of Materials Chemistry A, 2015, 3, 713-718.

[57] Yuan Y C, Sun Y X, Yan S J, Zhao J Q, Liu S M, Zhang M Q, Zhang X X, Jiang L. Multiply fully recyclable carbon fibre reinforced heat-resistant covalent thermosetting advanced composites. Nature Communications, 2017, 8, 1-11.

[58] Gu S L, Zhu L, Mercier C, Li Y J. Glass-fiber networks as an orbit for ions: fabrication of excellent antistatic PP/GF composites with extremely low organic salt loadings. ACS Applied Materials \& Interfaces, 2017, 9, 18305-18313.

[59] Luo W, Zhang Y, Xu S M, Dai J Q, Hitz E, Li Y J, Yang C P,
Chen C J, Liu B Y, Hu L B. Encapsulation of metallic Na in an electrically conductive host with porous channels as a highly stable Na metal anode. Nano Letters, 2017, 17, 3792-3797.

[60] Zhang K B, Zhang M M, Qiao J F, Zhang S L. Enhancement of the corrosion resistance of zinc-aluminum-chromium coating with cerium nitrate. Journal of Alloys and Compounds, 2016, 692, 460-464.

[61] Lv B M, Xie H, Xu R, Fan X L, Zhang W X, Wang T J. Effects of sintering and mixed oxide growth on the interface cracking of air-plasma-sprayed thermal barrier coating system at high temperature. Applied Surface Science, 2016, 360, 461-469.

[62] Darmanin T, Guittard F. Recent advances in the potential applications of bioinspired superhydrophobic materials. Journal of Materials Chemistry A, 2014, 2, 16319-16359. 